\title{
DESIGN OF A CENTRIFUGAL COMPRESSOR FOR NATURAL GAS
}

\author{
S. K. Kurauchi ${ }^{\mathrm{a}}$, \\ and J. R. Barbosa ${ }^{b}$ \\ ${ }^{a}$ Instituto Tecnológico de Aeronáutica \\ Divisão de Engenharia Mecânica \\ Departamento de Turbomáquinas \\ São José dos Campos, SP, Brasil \\ CEP. $12228-900$ \\ sandro.kurauchi@gmail.com \\ ${ }^{\mathrm{b}}$ Instituto Tecnológico de Aeronáutica \\ Divisão de Engenharia Mecânica \\ Departamento de Turbomáquinas \\ São José dos Campos, SP, Brasil \\ CEP. $12228-900$ \\ barbosa@ita.br \\ Received: October 25, 2013 \\ Revised: November 21, 2013 \\ Accepted: December 30, 2013 \\ ABSTRACT \\ This work presents the design of a centrifugal compressor for natural gas in \\ three steps. The first step is the 1-D preliminary design heavily based on \\ empirical data, only the design point was considered here. The second step \\ is the flow analysis in the meridional plane. The last step is the CFD \\ analysis to verify if the 1-D design methodology is adequate. The CFD \\ simulations showed good agreement with the results obtained in the \\ preliminary design, proving the importance of empirical data in the design \\ of centrifugal compressors. \\ Keywords: centrifugal compressor, CFD, turbomachinery.
}

\section{NOMENCLATURE}

b blade width

$\dot{\mathrm{m}}$ mass flow rate

LR impeller axial length

$\mathrm{M}$ Mach number

$\mathrm{N}$ rotational speed (rpm)

$\mathrm{R}$ radius from axis of rotation

$\mathrm{V}$ absolute velocity

$\mathrm{W}$ relative velocity

$\mathrm{R}$ impeller radius

$\mathrm{b}$ blade height

$\mathrm{L}$ diffuser length

$\mathrm{z}$ number of blades

\section{Greek Symbols}

$\alpha$ absolute flow angle from the tangential direction

$\beta$ relative flow angle from the tangential direction

$\beta_{\mathrm{b}}$ blade angle

E diffuser divergence angle

$\eta$ efficiency

$\lambda$ velocity ratio $\left(\mathrm{V}_{4} / \mathrm{V}_{2}\right)$

$\omega$ angular velocity $(\mathrm{rad} / \mathrm{s})$

\section{Subscripts}

1 impeller inlet

2 impeller outlet

3 diffuser inlet

4 diffuser outlet diff diffuser

i inner radius

o outer radius

$\mathrm{R}$ rotor

\section{INTRODUCTION}

Natural gas is used primary as fuel in industrial, commercial, residential and automotive market shares. According to Kidnay and Parrish (2006), coal and oil produce approximately 1.4 to 1.75 times the amount of dioxide of carbon $\left(\mathrm{CO}_{2}\right)$ and 5 times the amount of nitrogen oxides (NOx) that the natural gas burn produces. In the next decades, natural gas will produce more energy than all renewable energy sources together. Pipelines are one of the main means of transport for natural gas and compressors of the centrifugal type are the most used ones in these systems.

\section{DESIGN METHODOLOGY}

A thorough search for design methodologies indicated that most of the compressor design knowledge was described within the years 1970's. The process starts with the preliminary design, when 
it will be studied the ways a compressor can achieve the design duty with the best efficiency on a onedimensional basis. In this stage, the principal dimensions and angles of the compressor are calculated for the design point using $1 \mathrm{D}$ equations and relying on empirical data. The mechanical limitations of the materials are also taken in account during the preliminary design.

With the compressor dimensions, the hub and shroud meridional contours are drawn. Knowing the blade angles and the meridional shape it is possible to have a first estimate for the blade profile.

The flow in the impeller is then analyzed in a 2D basis. A hub-to-shroud and/or blade-to-blade aerodynamic analysis is performed and the meridional shape and blade profile can be improved. The drawback in this type of analysis is the fact that the flow is considered inviscid so the results are not good near walls, but are enough to foresee many problems like the possibility of flow separation, for example.

The last step is the $3 \mathrm{D}$ analysis. The compressor aerodynamics is analyzed through CFD techniques while, in parallel, the mechanical design is also being reviewed.

\section{Preliminary design}

For any compressor design, the following data must be known: mass flow rate $(\dot{\mathrm{m}})$, inlet conditions $\left(\mathrm{p}_{01}\right.$ and $\left.\mathrm{T}_{01}\right)$, pressure ratio and working fluid properties.

In this step it is assumed that the properties at each station have to be considered representative of the flow across the transversal sections, the velocity of the flow at the inlet of the impeller has the same direction as the axis of rotation and the axial velocity at the inlet is constant from hub to tip of the impeller.

A computer program based on the methodology developed by Vavra (1970) was used for the preliminary design. Some parameters must be assumed as input data.

1. The blade angle at the impeller exit $\beta_{2 b}$. The designer must choose the amount of backsweep based on the design requirements like the operating range, but the mechanical stresses must also be taken into account.

2. Ratio $\mathrm{R}_{10} / \mathrm{R}_{2}$, which cannot be higher than 0.70 to 0.75 (Vavra, 1970), otherwise the outer rotor contour curvature would not be small enough in the meridional plane.

3. Number of blades $z_{R}$.

4. The impeller rotational speed N. Usually it is a design requirement since the compressor will be driven by an electrical motor or gas turbine.

5 . The impeller tip radius $R_{2}$. It is chosen based on the available space for the machine, bearing in mind that the blade outlet height must be greater than a minimum value, here adopted as $0.08 \mathrm{R}_{2}$.

6. Flow outlet absolute angle $\alpha 2$. Came and
Robinson (1999) found the range of $17^{\circ} \leq \alpha_{2} \leq 21^{\circ}$ an acceptable limit.

7. Impeller efficiency, $\eta_{R}$. The desired rotor efficiency $\eta_{R}$ must also be used as input data.

8. Diffuser diffusion ratio $\lambda$. The ratio $\mathrm{V}_{4} / \mathrm{V}_{2}=\lambda$, which defines the diffusion ratio in the diffuser.

The preliminary computer program also places some constraints for a solution.

- It is desirable to minimize $\mathrm{M}_{\mathrm{w} 1}$ to avoid shock losses or the choke condition. In high flow impellers the flow reaches transonic velocities at the inlet but the local shock losses are relatively small up to $\mathrm{M}_{\mathrm{w} 1}=1.3$ (Dalbert et al., 1999).

- The desirable isentropic efficiency $\eta_{\mathrm{c}}$.

- A maximum value for $\mathrm{M}_{\mathrm{v} 2}$, because the design point can be near the choke region if the flow reaches the sonic velocity.

- The ratio $\mathrm{W}_{2} / \mathrm{W}_{10}$ between 0.45 and 0.6 (Came and Robinson, 1999).

- According to Vavra (1970), the ratio $b_{2} / R_{2}$ has influence on the disk friction losses in the impeller and experience shows that its value should not be less than 0.08 .

- The degree of reaction represents the amount of enthalpy rise in the impeller in relation to the enthalpy rise in the stage. According to Dalbert et al. (1999), typical values are about 0.6-0.7.

- The ratio $R_{1 i} / R_{10}$ cannot be too small; otherwise, there will not be enough space for a sufficient number of blades with adequate thickness (Vavra, 1970).

- $\mathrm{U}_{2}$, because of the material stress limits. The maximum speed for cast aluminum is $200-300 \mathrm{~m} / \mathrm{s}$, for forged machine aluminum is $500 \mathrm{~m} / \mathrm{s}$ and for titanium is around $650-700 \mathrm{~m} / \mathrm{s}$ (Came and Robinson, 1999).

\section{analysis}

Two-dimensional meridional plane flow

This study was carried out to detect possible flow separations, assuming that large relative velocities gradients may cause flow separations. The basis for the study is one of Katsanis' works, which predicts inviscid properties along the impeller channel.

Katsanis (1964) developed a method and a computer program that calculates a two-dimensional inviscid solution for the velocity and pressure distribution along the meridional plane. This computer program, after introduction of modifications to make it usable, made at the Center for Reference on Gas Turbines, was used as a basis for the calculations. The meridional plane is the projection of a mean stream surface between two blades in a plane that contains the axis of rotation. It is assumed that the meridional plane and the inlet total conditions are known.

The method uses streamlines and a set of arbitrary fixed curves from hub to shroud, called 
quasi-orthogonals, to establish a grid for the solution.

The machine to be designed is a single-stage centrifugal compressor with unshrouded impeller and island diffuser. Although a vaneless diffuser is more common for pipeline applications, a vaned one was chosen for a better efficiency.

For the compressor design, the following values were established:

Mass flow rate:

Pressure ratio:

Inlet stagnation pressure:

Inlet stagnation temperature:

$307 \mathrm{~K}$

The gas type must also be considered. Table 1 shows the gas composition used in this particular design.

Table 1. Natural gas composition used in the design.

\begin{tabular}{lcc}
\hline Gas & Formula & Volume (\%) \\
\hline Methane & $\mathrm{CH}_{4}$ & 89 \\
Ethane & $\mathrm{C}_{2} \mathrm{H}_{6}$ & 6 \\
Propane & $\mathrm{C}_{3} \mathrm{H} 8$ & 1.8 \\
iso-Butane & $\mathrm{C} 4 \mathrm{H}_{10}$ & 1 \\
Carbon dioxide & $\mathrm{CO}_{2}$ & 1.5 \\
Nitrogen & $\mathrm{N}_{2}$ & 0.7
\end{tabular}

\section{RESULTS AND DISCUSSION}

Using the preliminary design program the principal dimensions and angles of a compressor stage were computed, as shown in Tab. 2 and Tab. 3.

Using a simple method to build the meridional channel of the compressor, the meanline of the meridional channel was considered a quarter of an ellipse, linking the inlet to the outlet of the impeller. Both at the inlet and at outlet the meanline makes an angle of $90^{\circ}$ with the leading and trailing edges. The hub and shroud contours were defined varying the cross sectional area linearly from inlet to outlet. A sketch of the meridional channel and grid, and the calculated meridional channel are shown in Fig. 1.

Table 2. Calculated geometrical parameters.

\begin{tabular}{lll}
\hline Parameter & Dim. & Value \\
\hline $\mathrm{R}_{2}$ & $(\mathrm{~m})$ & 0.160 \\
$\mathrm{R}_{1 \mathrm{o}}$ & $(\mathrm{m})$ & 0.096 \\
$\mathrm{R}_{1 \mathrm{i}}$ & $(\mathrm{m})$ & 0.0648 \\
$\mathrm{~b}_{2}$ & $(\mathrm{~m})$ & 0.0171 \\
$\mathrm{R}_{3}$ & $(\mathrm{~m})$ & 0.1712 \\
$\mathrm{R}_{4}$ & $(\mathrm{~m})$ & 0.2439 \\
$\mathrm{~L}_{\mathrm{R}}$ & $(\mathrm{m})$ & 0.0637 \\
$\mathrm{z}_{\mathrm{R}}$ & & 15 \\
$\mathrm{z}_{\text {diff }}$ & & 14 \\
\hline
\end{tabular}

Table 3. Calculated angles.

\begin{tabular}{lcl}
\hline Parameter & - & Value \\
\hline$\alpha_{1}$ & $\left({ }^{\circ}\right)$ & 90 \\
$\alpha_{2}$ & $\left({ }^{\circ}\right)$ & 20 \\
$\alpha_{3}$ & $\left({ }^{\circ}\right)$ & 20 \\
$\beta_{10}$ & $\left({ }^{\circ}\right)$ & 30 \\
$\beta_{1 \mathrm{i}}$ & $\left({ }^{\circ}\right.$ & 40.54 \\
$\beta_{2}$ & $\left({ }^{\circ}\right)$ & 47.68 \\
\hline
\end{tabular}

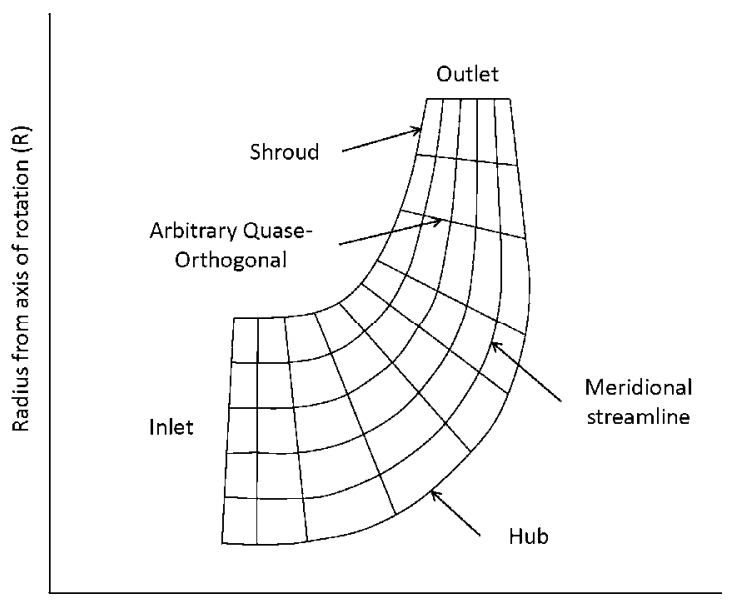

Axial coordinate $(z)$

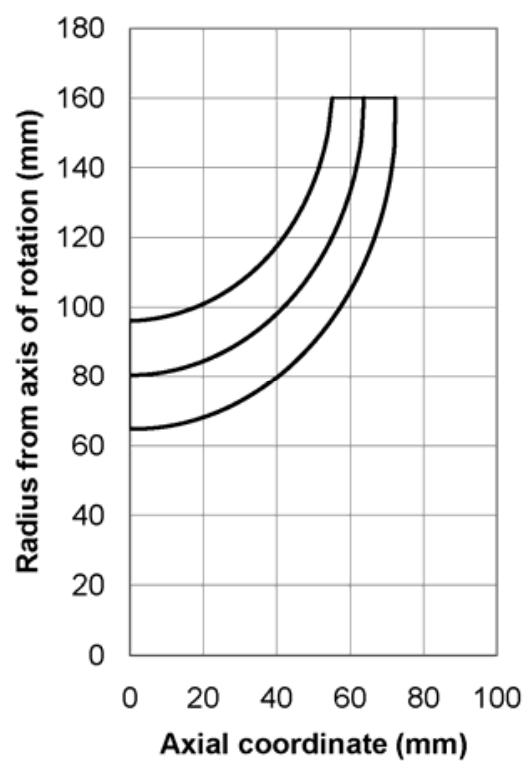

Figure 1. Meridional channel schematics and meridional channel as calculated.

The velocities distribution computed by the program can be seen in Fig. 2. At the inlet, there is flow acceleration because the gas reaches the leading edge, which causes a decrease in the flow area. It is noticeable a decreasing velocity near the hub, after which the flow accelerates indicating a high probability of boundary layer separation. 


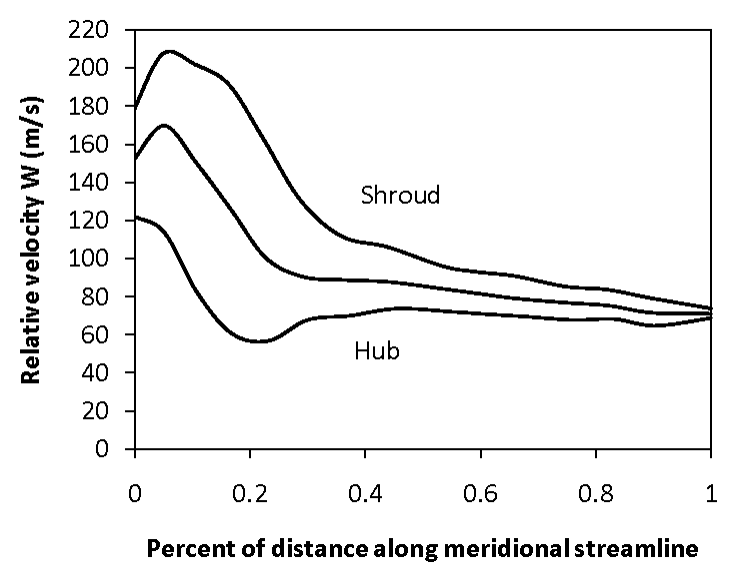

Figure 2. Relative velocities along the meridional plane.

In order to get a better velocities distribution, a modification in the original blade contour was done. In the hub, where the flow had an undesirable behavior, the gas path was modified to have a smoother change of direction. The red line represents the original blade contour in Fig. 3, whereas the black line is the modified contour.

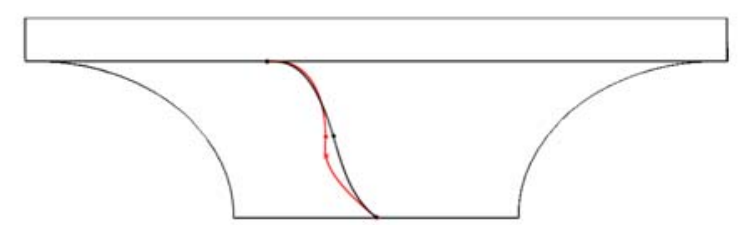

Figure 3. Modification in the blade geometry.

The same procedure was repeated to analyze the flow in the meridional channel with the new impeller blades. The new velocities distribution is shown in Fig. 4. Only visual analysis of the relative velocity curve along the hub, mid-height and tip was used to choose an adequate flow passage.

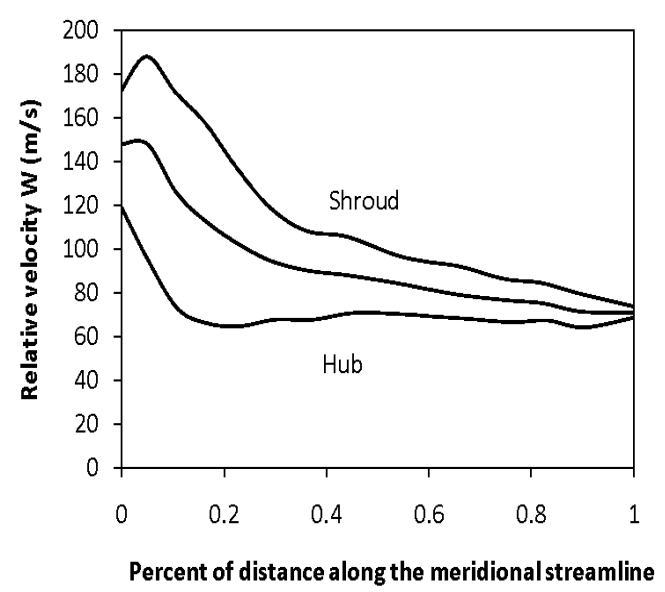

Figure 4. Relative velocities along the meridional plane in the modified impeller.
The new blades produce a different velocity distribution near the hub. The undesirable flow acceleration was practically eliminated. It means that there is less probability of flow separation. Additionally, in the shroud, where there are the highest deceleration ratios, the new curve is smoother than before. The compressor stage modeled with Solid Works 2010 can be seen in Fig. 5.

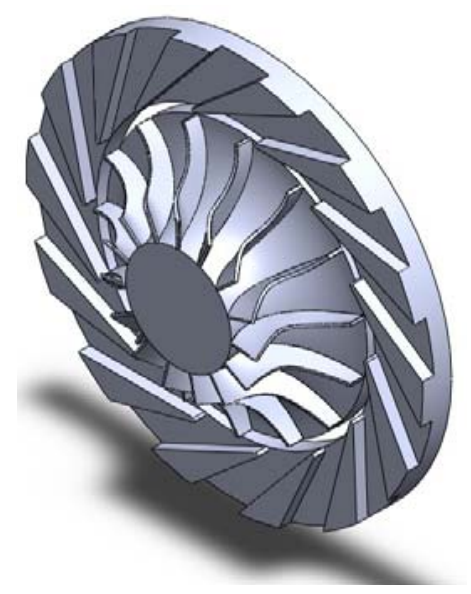

Figure 5. Impeller and diffuser assembled.

\section{CFD simulations}

The software used was ANSYS CFX 13. In this analysis, the fluid was treated as a real gas using the Peng Robinson equation of state. The flow was considered steady. Only one passage channel in the impeller and one in the diffuser were simulated by setting a condition of rotational periodicity. The time step used was $1 / \omega$. The turbulence model used was the SST. The discretization scheme used was the upwind scheme with a specified blend factor of 0.80 .

The mesh (Fig. 6) created for the compressor stage is of the tetrahedrical type, with 296,390 nodes and $1,458,559$ elements. In this work, the tip clearance was not considered.

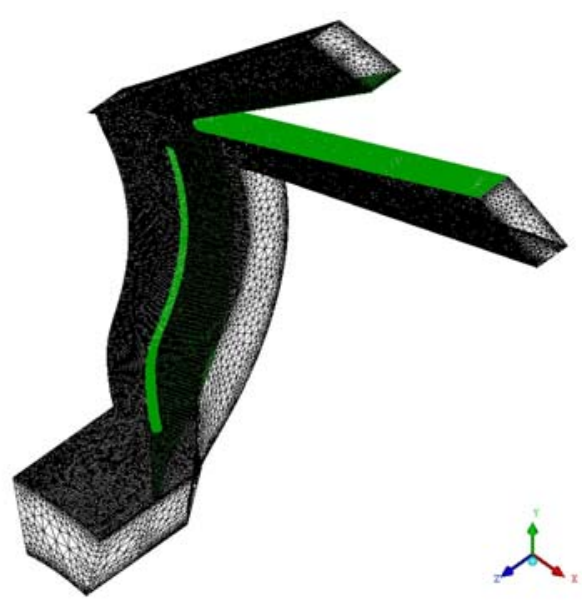

Figure 6. The mesh. 
The boundary conditions used initially were taken from the preliminary design:

Inlet stagnation pressure ( $\mathrm{p} 01)$ : $6.48 \mathrm{MPa}$

Inlet stagnation temperature (T01):

$307 \mathrm{~K}$

Outlet static pressure (p4):

$9.55 \mathrm{MPa}$ vectors.

Figure 7 shows the simulated stage velocity

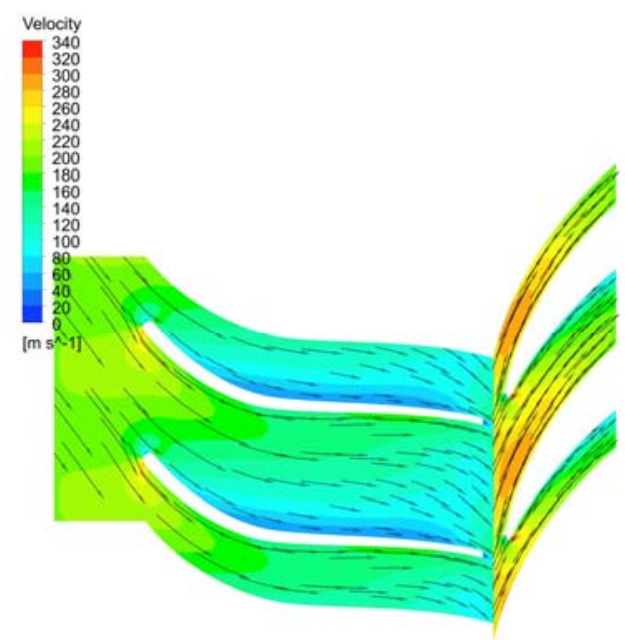

Figure 7 . Velocity vectors at $50 \%$ of blade height.

After analysis of the CFD results, it was concluded that the geometry was adequate and the compressor would perform as specified.

Based on this conclusion, two lines of constant rotational speeds $(100 \%$ and $80 \%)$ were calculated aiming at assembling the compressor map. For the $100 \%$ rotation speed, 14 points were analyzed and, for the $80 \%$ rotational speed, 8 points.

Figure 8 shows the variation of total pressure ratio for different mass flow rates. As expected, a reduced speed implied in a reduced pressure ratio since part of the enthalpy rise is caused by the term $\frac{1}{2}\left(U_{2}^{2}-U_{1}^{2}\right)$. At design rotational speed $N$, the compressor could achieve a pressure ratio equal to 1.63 while at $80 \% \mathrm{~N}$, the maximum pressure ratio was 1.37 .

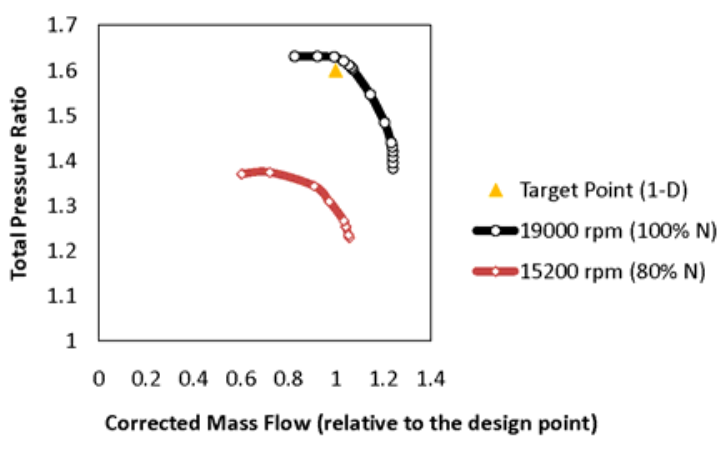

Figure 8. Total pressure ratio $\mathrm{x}$ corrected mass flow.
When the flow is approaching the choke condition, the stage performance degrades rapidly and consequently the outlet pressure decreases with the efficiency as shown in Fig. 9. The incidence angle varies with the change in mass flow rate, being a source of losses. The flow reaches the diffuser with a different angle from the one it was designed for. Moreover, the internal friction losses also increase with the mass flow rate.

The target points are the ones computed by the preliminary design program. For the same mass flow, the results from the CFD simulations showed that the compressor could achieve a pressure ratio higher than the value calculated in the original design. The overall results show a good agreement between the 1D method results and the CFD simulations.

For the design speed, the simulations showed that choke occurs at a mass flow rate of about $93 \mathrm{~kg} / \mathrm{s}$ and at $80 \%$ of the design speed; choke occurs at 79 $\mathrm{kg} / \mathrm{s}$. On the other side of the curves, it is hard to say when surge starts. In the present work, surge limit is considered the point where the slope is zero.

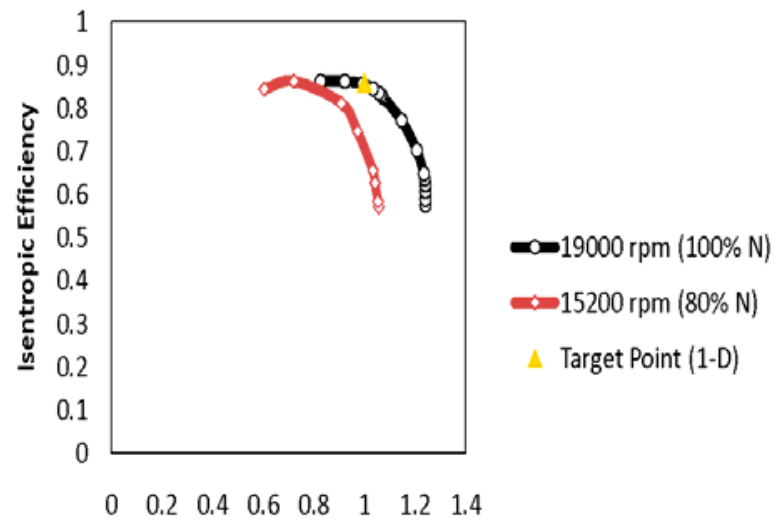

Corrected Mass Flow (relative to the design point)

Figure 9. Stage isentropic efficiency $\mathrm{x}$ corrected mass flow.

\section{CONCLUSIONS}

Nowadays CFD techniques are very accurate, however it is not easy to tell how close from the reality the simulations are without proper tests with a prototype. However, the simulations results can be used as reference for the compressor stage design.

The point of departure for the centrifugal compressor design was appropriately selected, following Vavra's suggestions. Looking at the compressor performance curves, it is possible to conclude that the empirical data used in the preliminary design were correctly chosen, so that the design became closer to the optimum, a very good point of departure for future optimization.

Overall, the CFD simulations showed that the preliminary design program produced good results. 


\section{ACKNOWLEDGEMENTS}

FINEP (Financiadora de Estudos e Projetos), TGM Turbinas, IAE (Instituto de Aeronáutica e Espaço), FAPESP (Fundação de Apoio à Pesquisa do Estado de São Paulo), CNPq (Conselho Nacional de Desenvolvimento Científico e Tecnológico), CAPES (Coordenação do Aperfeiçoamento do Pessoal de Nível Superior) and ANEEL (Agência Nacional de Energia Elétrica) are acknowledged for their support to the research carried out at the Center for Referennce on Gas Turbines (ITA).

\section{REFERENCES}

Came, P. M., and Robinson, C. J., 1999, Centrifugal Compressor Design, Proceedings of the Institution of Mechanical Engineers, Part C: Journal of Mechanical Engineering Science, Vol. 213, pp. 139-155.

Dalbert, P., Ribi, B., Kmeci, T., and Casey, M., 1999, Radial Compressor Design for Industrial Compressors, Proceedings of the Institution of Mechanical Engineers, Part C: Journal of Mechanical Engineering Science, Vol. 216, pp. 71-83.

Katsanis, T., 1964, Use of Arbitrary QuasiOrthogonals for Calculating flow Distribution in the Meridional Plane of a Turbomachine, NASA TN D2546.

Kidnay, A. J. and Parrish, W., 2006, Fundamentals of Natural Gas Processing, CRC Press.

Vavra, M., 1970, Basic Elements for Advanced Design of Radial-flow Compressors, AGARD Lecture Series, Vol. 39, pp. 6-11. 